\title{
An ecological cognitive semantics
}

\section{SIMON UFFE BORCHMANN}

The starting point of the article is that the established linguistic descriptions of messages are neither accurate nor informative when applied to messages that form part of performing certain activities. The problem is traced back to the semantic and pragmatic basis of the established descriptions. To solve the problem a semantics based on Gibson's theory of affordances is suggested. The pragmatic assumption that forms the basis of the alternative semantics is that we use messages to share values, that is, information about affordances. Two basic semantic structures are proposed. The first is a relationship between an indication of a value, for example, southeast, and an identification of the mode of differentiation to be used in the understanding of the indicated value, for example, wind direction. The other is a relation between an indication of a value, for example, southeast, and an indication of the precondition for the indicated value, e.g., in the evening. The claim is that these two structures regulate the formation of messages. This alternative semantics enables an accurate characterization and informative analysis of messages that form part of performing certain activities. 